\title{
Effect of bisphenol A on the EGFR-STAT3 pathway in MCF-7 breast cancer cells
}

\author{
WEI ZHANG ${ }^{1,2}$, YANQIU FANG $^{3}, \mathrm{XU} \mathrm{SHI}^{4}$, MINGLEI ZHANG $^{5}, \mathrm{XIAOQI} \mathrm{WANG}^{6}$ and YAN TAN $^{7,8}$ \\ ${ }^{1}$ Research Center, The First Clinical Hospital of Jilin University; ${ }^{2}$ Department of Obstetrics and Gynecology, \\ Nursing College of Jilin University; ${ }^{3}$ Department of Tumor Biological Therapy, Jilin Provincial Hospital; \\ ${ }^{4}$ Virus and AIDS Research Center, The First Clinical Hospital of Jilin University, Changchun, Jilin 130021; \\ ${ }^{5}$ Department of Orthopaedics, China-Japan Union Hospital of Jilin University, Changchun, Jilin 130033, P.R. China; \\ ${ }^{6}$ Division of Hematology/Oncology, Herbert Irving Comprehensive Cancer Center, Columbia University Medical Center, \\ New York, NY, USA; ${ }^{7}$ Research Center, The First Clinical Hospital of Jilin University; \\ ${ }^{8}$ Department of Tumor Biological Therapy, Jilin Provincial Hospital, Changchun, Jilin 130021, P.R. China
}

Received June 3, 2011; Accepted August 30, 2011

DOI: $10.3892 / \mathrm{mmr} .2011 .583$

\begin{abstract}
The aim of this study was to explore the effect of bisphenol A (BPA) on the EGFR-STAT3 pathway in breast cancer. We applied 3-(4,5-dimethylthiazol-2-yl)2,5-diphenyl tetrazolium bromide (MTT) cytotoxicity assay to the analysis of the responsiveness of MCF-7 cells to BPA. Gene expression was assayed at the transcriptional and translational levels by reverse transcription-PCR and Western blotting. We explored the effects of BPA on MCF-7 cell proliferation through inhibition of the related genes, STAT3, using RNA interference, and EGFR, using its inhibitor AG1478. The optimal concentration and time point of BPA-induced proliferation in MCF-7 cells are $1 \mu \mathrm{M}$ and $24 \mathrm{~h}$, respectively. BPA significantly increased the expression of STAT3 at a concentration of $1 \mu \mathrm{M}$ following treatment for $48 \mathrm{~h}$ and the expression of STAT3 was downregulated after blocking EGFR. When STAT3 was blocked in MCF-7 cells, BPA did not appear to induce cell proliferation. Treatment with BPA $(1 \mu \mathrm{M})$ in the presence of AG1478 for $48 \mathrm{~h}$ resulted in the stimulation of cell growth in MCF-7 cells, similar to that of the BPA alone treatment. BPA increases STAT3 expression, which is a major factor in the pathway of BPA-induced proliferation, and STAT3 activation contributes to BPA-induced breast cancer cell proliferation. However,
\end{abstract}

Correspondence to: Professor Yan Tan, Research Center, The First Clinical Hospital of Jilin University; Department of Tumor Biological Therapy, Jilin Provincial Hospital, Changchun, Jilin 130021, P.R. China

E-mail: tanyan49@hotmail.com

Professor Xiaoqi Wang, Division of Hematology/Oncology, Herbert Irving Comprehensive Cancer Center, Columbia University Medical Center, New York, NY, USA

E-mail: xw2200@columbia.edu

Key words: breast cancer, bisphenol A, EGFR, STAT3, RNA interference
EGFR mediates negative signaling for BPA-induced breast cancer cell proliferation.

\section{Introduction}

Breast cancer has the highest incidence of female neoplastic diseases and sporadic breast cancer is the most common cancer diagnosed in American and Northern European women (1). The occurrence and development of breast cancer is a hormone-dependent and multi-step process, which may be induced by various factors such as hormones, chemicals, radiation, viruses and genetic factors, and according to certain reports, by environmental factors as well (2). Certain environmental chemicals may simulate estrogens completely or partly by binding to their receptors.

Savis et al hypothesized that environmental estrogens (EEs) are likely to be related to breast and ovarian cancer (3). Bisphenol A (BPA) was shown to be an EE and is widely used in plastic products, including food and water containers, baby bottles, linings of metal food and beverage cans, medical tubing, epoxy resins and dental fillings (4). Neonatal exposure to BPA is associated with higher body weight, increased breast and prostate cancer incidence and altered reproductive function (5). Experimental evidence indicates that exposure to BPA during childhood increases the risk of breast cancer in the adult female (6). As a consequence of this widespread opportunity for exposure, $95 \%$ of Americans carry detectable levels of BPA (7), which is currently one of the highest volume chemicals produced in the world (8). BPA may be digested and absorbed in the human body and increases the actual loading doses of estrogen (4), thereby increasing the incidence of breast, ovarian and endometrial cancer.

The mechanism by which BPA exerts its biological actions remains to be determined. The majority of studies have examined the manner in which BPA stimulates breast cancer cell proliferation and the related research on BPA is focused on estrogen receptors (ERs) (9), chemoresistance (10) and the apoptosis proteins $\mathrm{Bcl}-2$ and $\mathrm{Bax}(11)$. However, the potential 
effect of BPA on signal pathways have received little attention. Few studies investigating BPA signal pathways exist. Park et al (12) have reported that EE-activated ERK1/2 or p38 is unlikely to be involved in cell growth in estrogen-responsive ovarian cancer cells. Certain studies have confirmed the effectiveness of the activated EGFR-STAT3 pathway in breast cancer (13) and the cooperated efforts of STAT3 and nuclear EGFR of controlling cell transcription (14). EGFR is involved in a variety of downstream signaling pathways including MEK >ERK, PI3K>AKT, STAT, p38 and JNK $(15,16)$. It has been suggested that the JAK/STAT pathway is one of the significant downstream pathways for EGFR signaling (13). In addition, the existence of EGFR-independent signaling for STAT3 activation has been reported, enhancing the importance of STAT3 activation in tumor growth (17). Therefore, in this study, we inhibited the expression of STAT3 with RNA interference, and of EGFR with the highly selective Tyrphostin AG1478 in breast cancer MCF-7 cells, to explore the effect of BPA on the EGFR-STAT3 signaling pathway and its mechanism of carcinogenesis.

\section{Materials and methods}

Cells, drugs and antibody. The human breast cancer MCF-7 and MDA-MB-231 cells were purchased from ATCC (Manassas, VA, USA). Phenol red-free 1640 medium, RPMI-1640, Opti-MEM medium, fetal bovine serum (FBS) and pancreatic enzyme were purchased from Gibco (Invitrogen, Carlsbad, CA, USA): BPA was kindly provided by Dr Huang (Jilin University): Tyrophostin AG1478 was purchased from Shanghai Baijin Biological Co. (China), and Lipofectamine 2000 transfection reagent was purchased from Invitrogen: $\beta$-actin, STAT3 (sc-482) antibody was purchased from Santa Cruz Biotechnology (Santa Cruz, CA, USA).

Design and synthesis of STAT3 siRNA. The siRNA design and synthesis service was provided by GenePharma, Shanghai Co. (China). According to a series of Genbank accession numbers, the sequence information of the eight sites selected in designing siRNA includes: siRNA1, STAT3-homo-683 (GCA GGAUCUA GA ACAGAAAT T); siRNA2, STAT3-homo-1148 (GGAGCUGUUUAGAAACUUATT); siRNA3, STAT3-homo-376 (CCACUUUGGUGU UUCAUAATT); siRNA4, STAT3-homo-1244 (CGUCCA GUUCACUACUAAATT); siRNA5, STAT3-homo-1358 (CCCGGAA A UUUA A C AUUCUTT); siRNA6, STAT3-homo-1815 (GGGACCUGGUGUGAAUUAUTT); siRNA7, STAT3-homo-1964 (GGUACAUCAUGG GCUUUAUTT); siRNA8, STAT3-homo-2422 (GAU ACGACUGAGGCGCCUATT).

Cell culture. MCF-7 and MDA-MB-231 (control group) cells were routinely maintained and grown in RPMI-1640 medium supplemented with $10 \% \mathrm{FBS}$ at $37^{\circ} \mathrm{C}$ in a humidified atmosphere of $5 \% \mathrm{CO}_{2}$ in an incubator and in phenol red-free 1640 medium for 4 days to exhaust the phenol red prior to initiating the experiment. Cells were seeded in 96-well plates at a density of $3 \times 10^{3}$ cells/well and were continued to be cultured by $24 \mathrm{~h}$ for 3-(4,5-dimethylthiazol-2-yl)2,5-diphenyl tetrazolium bromide (MTT) assay.
Cell proliferation assay. BPA was dissolved in dehydrated alcohol at stock concentrations of $10 \mathrm{mM}$ and was diluted to a working concentration of $1,000 \mathrm{nmol} / 1$ with phenol red-free 1640 medium prior to treatment. BPA was then 10-fold diluted into $10^{-1}-10^{5} \mathrm{nM}$. MCF-7 and MDA-MB-231 cells were plated at a density of 6,000 and 8,000 cells/well, respectively, in 96-well plates in plating medium, with the setting of 5 repetitive samples for each dose and the dehydrated alcohol group as the control group. Cells were treated with BPA for 24, 48 and $72 \mathrm{~h}$, respectively, and changes in proliferation relative to vehicle treatment were examined by MTT assay. MTT was added at a final concentration of $0.5 \mathrm{mg} / \mathrm{ml}$ for $2 \mathrm{~h}$. Following medium aspiration, the formazan dye was extracted with DMSO and absorbance at $490 \mathrm{~nm}$ was determined for each well with a microplate reader. Measured counts were regarded as relative indices of proliferation by normalizing to untreated cell controls.

Quantitative reverse transcription-PCR assay. Total RNA was isolated using TRIzol and cDNA was synthesized (SuperScript III reverse transcriptase) with the use of oligo (dT) primers from Invitrogen. Samples were run in triplicate on Stratagene M x 2005P (Agilent, Santa Clara, CA, USA), using the following variables: denaturation at $95^{\circ} \mathrm{C}$ for $3 \mathrm{~min}$ followed by 40 cycles of amplification $\left(95^{\circ} \mathrm{C}\right.$ for $30 \mathrm{sec}$ and $62^{\circ} \mathrm{C}$ for $40 \mathrm{sec})$. We applied Primer Premier 5 software to design the STAT3 primer. The relative expression levels of STAT3 were normalized with a GAPDH housekeeping gene as the internal control. Primers for PCR amplification were: STAT3, forward: 5'-TCCATCAGCTCTACAGTGACAGC-3' and reverse: 5'-TCCCAGGAGATTATGAAACACC-3'; GAPDH, forward: 5'-CATGAGAAGTATGACAACAGCCT-3' and reverse 5'-AGTCCTTCCACGATACCAAAGT-3'.

Immunoblotting. MCF-7 cells were harvested, washed twice with cold phosphate-buffered saline (PBS), and then lysed with lysis buffer [PBS containing 1\% Triton X-100, complete protease inhibitor cocktail (Roche, Germany) and RNase inhibitor (New England Biolabs, Ipswich, MA, USA)] at $4^{\circ} \mathrm{C}$ for $30 \mathrm{~min}$. The protein concentration was determined using the Pierce (Rockford, IL, USA) bicinchoninic acid (BCA) protein assay. We added $5 \mu 12 \mathrm{X}$ SDS-PAGE loading buffer to $80 \mu \mathrm{g}$ protein/well, and the samples were then electrophoresed in $10 \%$ SDS-PAGE minigels. We used a PVDF membrane to transfer the proteins in transfer buffer $[25 \mathrm{mM}$ Tris base, 0.2 M glycine and 20\% methanol ( $\mathrm{pH} 8.5)]$. After being transferred to the PVDF membrane, samples were sealed in blocking buffer (1X TBS, $0.1 \%$ Tween-20 and 5\% w/v non-fat dry milk) for $1 \mathrm{~h}$ and incubated overnight with mouse monoclonal antibody to STAT3 (1:2000). Following incubation with goat anti-rabbit secondary antibody (1:1000), detection was performed using SuperSignal chemiluminescence reagents (Pierce).

Small interfering RNA knockdown. Knockdown of STAT3 was performed using specific small interfering RNA (siRNA) pools targeting STAT3 purchased from GenePharma, Shanghai Co. (China). Cells were divided into mock, blank (normal cells) and negative control groups. Introduction of siRNA was performed with Lipofectamine 2000 according 
A

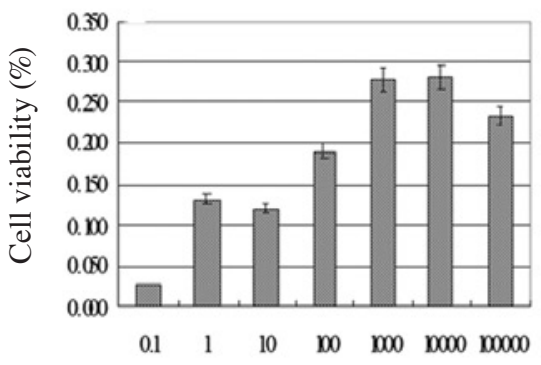

Concentration of BPA (nM)
B

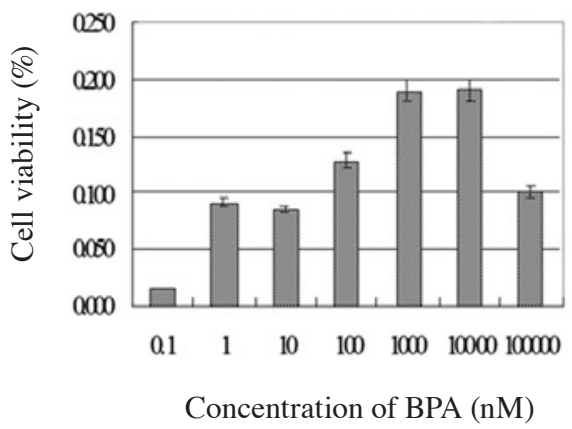

C

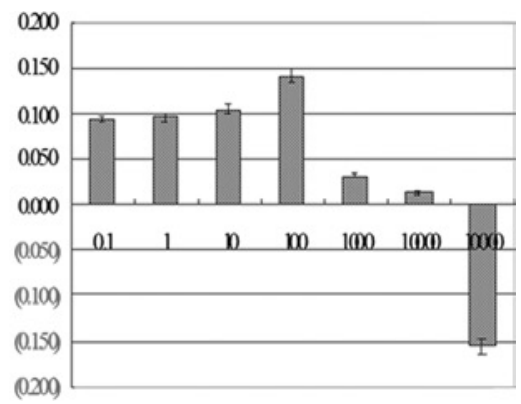

Concentration of BPA $(\mathrm{nM})$

Figure 1. MCF-7 cells were treated with BPA for 24,48 and $72 \mathrm{~h}$, followed by increasing BPA concentrations from $10^{-1}$ to $10^{5} \mathrm{nM}$. Cytotoxicity was determined by the MTT assay. (A-C) Effect of BPA-induced proliferation following treatment for 24, 48 and $72 \mathrm{~h}$, respectively. The relative proliferation rates of the BPA treatment group are shown as the percentage of the dehydrated alcohol group.

to the manufacturer's instructions (Invitrogen). Levels of STAT3 knockdown with different segments were assessed by RT-PCR and immunoblot analysis in pools of transfected cells.

STAT3 siRNA5 interference efficiency assay. MCF-7 cells were seeded in 6 -well plates at $1 \times 10^{6}$ cells/well in plating medium. Cells were washed with D-Hank's balanced solution and starved with phenol red-free 1640 medium without serum. For the transfection preparation we added $250 \mu 1$ Opti-MEM I and 200 pmol STAT3 siRNA5 to a $1.5 \mathrm{ml}$ EP tube (group A), and then $250 \mu \mathrm{l}$ Opti-MEM I and $5 \mu \mathrm{l}$ Lipofectamine 2000 to another $1.5 \mathrm{ml}$ EP tube (group B). We combined groups A and $\mathrm{B}$, and after placing them at room temperature for $5 \mathrm{~min}$ we mixed them, and placed the mixture (group E) at room temperature for 20-30 min. Group E was added to 6-well plates at $500 \mu \mathrm{l} /$ well blending them gently, and the cells were incubated for 4-6 h. Cells were treated with $1 \mu \mathrm{M}$ BPA after the transfection reagent was discarded. The cells were then incubated at $37^{\circ} \mathrm{C}$ in a humidified atmosphere of $5 \% \mathrm{CO}_{2}$ for $48 \mathrm{~h}$. The cells were collected for RT-PCR and Western blot analysis of STAT3.

Cell proliferation assay following STAT3 siRNA5 and BPA treatment. MCF-7 cells were seeded in 96-well plates at 5000 cells/well in plating medium, washed with D-Hank's balanced solution, and starved with phenol red-free 1640 medium without serum. Transfection was prepared under the following conditions: $125 \mu \mathrm{l}$ (5 repeats x $25 \mu \mathrm{l}$ ) Opti-MEM $\mathrm{I}$ and $50 \mathrm{pmol}(5$ repeats $\mathrm{x} 10 \mathrm{pmol})$ STAT3 siRNA5 were added to a $1.5 \mathrm{ml}$ EP tube (group C). Then $125 \mu \mathrm{l}(5$ repeats $\mathrm{x} 25 \mu \mathrm{l})$ Opti-MEM I and $1.25 \mu \mathrm{l}(5$ repeats $\mathrm{x} 0.25 \mu \mathrm{l})$ Lipofectamine 2000 were added to another $1.5 \mathrm{ml} \mathrm{EP}$ tube (group D). We blended groups $\mathrm{C}$ and D respectively, and mixed them after placing them at room temperature for $5 \mathrm{~min}$. The mixture (group F) was placed at room temperature for 20-30 min. Group F was added to 96 -well plates at $25 \mu \mathrm{l} /$ well and blended gently, and cells were incubated for 4-6 h. Cells were treated with $1 \mu \mathrm{M}$ BPA after the transfection reagent was discarded. The cells were then incubated at $37^{\circ} \mathrm{C}$ in a humidified atmosphere of $5 \% \mathrm{CO}_{2}$ in an incubator for $48 \mathrm{~h}$. Cells in 96-well plates were collected to determine cytotoxicity using the MTT assay, and divided into 6 groups: blank,
STAT3 siRNA5, BPA + dehydrated alcohol + STAT3 siRNA5, BPA + dehydrated alcohol + negative control, dehydrated alcohol + STAT3 siRNA5 and negative control.

Tyrphostin AG1478 interference test. A stock solution of AG1478 was produced in DMSO at $20 \mathrm{mM} / \mathrm{l}$ and then diluted with phenol red-free 1640 medium to a final working solution at $10 \mu \mathrm{M}$. MCF-7 cells were seeded in 6-well plates at $1 \times 10^{6}$ cells/well and 96-well plates at 5000 cells/well. BPA and AG1478 were added at the working concentrations, respectively, to 96-well plates with five repeats for each group. MTT cell growth assays were performed $48 \mathrm{~h}$ following the addition of drugs. After cells in 6-well plates were incubated for $24 \mathrm{~h}$, they were treated with AG1478 at the working concentration, and $48 \mathrm{~h}$ later were collected for STAT3 real-time RNA and Western blot assay.

Statistical analysis. Statistical analyses were conducted using SPSS software (version 11.5), with a single factor analysis of variance and independent samples t-test. Statistical significance was set at $\mathrm{P}<0.05$.

\section{Results}

Promoting cell proliferation by BPA in a dose-dependent manner. To determine the effect of BPA on cell proliferation, MCF-7 and MDA-MB-231 (control group) cells were treated with BPA to determine the optimal concentration and application time. Cytotoxicity was determined by the MTT assay following treatment with BPA for 24, 48 and $72 \mathrm{~h}$. Cells were treated with either vehicle or $1 \mu \mathrm{M}$ BPA and incubated for $24 \mathrm{~h}$ resulting in a significant increase of proliferation (Fig. 1A), which continued to the time point at $48 \mathrm{~h}$ (Fig. 1B). As demonstrated in Fig. 1C there was no effect on proliferation when treated with $1 \mu \mathrm{M}$ BPA for the 72-h treatment. Treatment with $100 \mu \mathrm{M}$ BPA showed a strong cytotoxic effect, although there was no significant effect of BPA-induced proliferation on MDA-MB-231 cells (Fig. 1).

Effect of BPA on the activation of STAT3. To investigate the effect of BPA on STAT3 activation, MCF-7 cells were treated with $1 \mu \mathrm{M}$ BPA for 24, 48 and $72 \mathrm{~h}$, respectively. According to the results of Western blotting, as demonstrated in Fig. 2, 


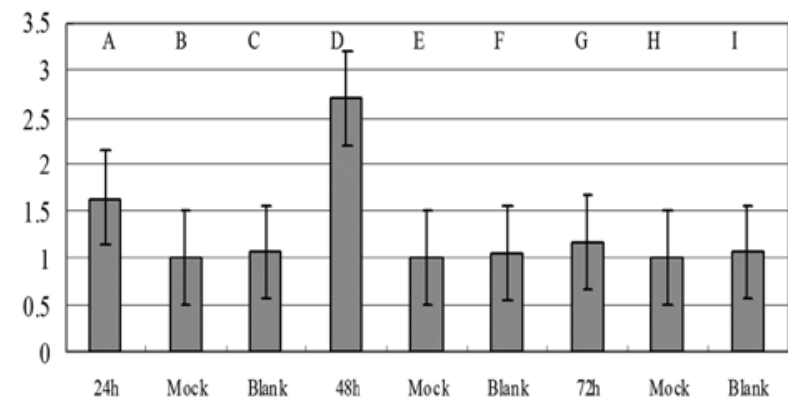

STAT3 expression relative to actin to mock

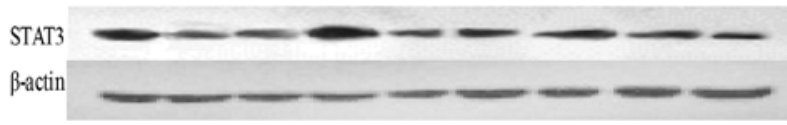

Figure 2. MCF-7 cells were treated with BPA and subsequently collected for STAT3 assay of Western blot analysis. Samples A, D and G are the expression of STAT3 following BPA treatment for 24, 48 and $72 \mathrm{~h}$, respectively. Samples $\mathrm{B}, \mathrm{E}$ and $\mathrm{H}$ show the expression of STAT3 of the mock group after being treated for 24,48 and $72 \mathrm{~h}$, respectively. Samples C, F and I show the expression of STAT3 of the blank group after being treated for 24,48 and $72 \mathrm{~h}$, respectively.

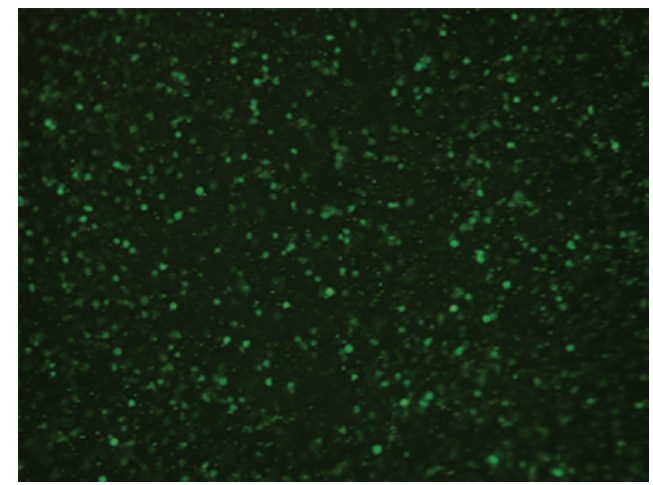

Figure 3. MCF-7 cells with NC-FAM were transfected in fluorescent light (magnification, x200). Transfection efficiency is determined by NC-siRNA with FAM labeled through cell counting under a fluorescence microscope.

the expression of STAT3 was significantly up-regulated with $1 \mu \mathrm{M}$ BPA treatment for $48 \mathrm{~h}$ (Fig. 2).

Overview of the transfection efficiency. As shown in Fig. 3 MCF-7 cells were transfected with FAM-labeled NC-siRNA and monitored with a fluorescent microscope (magnification, $\mathrm{x} 200$ ). The transfection efficiency was $80 \%$, which was determined by counting the percentage of fluorescence-bearing cells (Fig. 3).

Effective fragment of the screening results. To achieve effective RNA interference, we transfected MCF-7 cells with 8 STAT3 interference segments. As shown in Fig. 4, after combining the results of RT-PCR and Western blotting, we selected the 5th oligo (siRNA5) to carry out the subsequent experiments (Fig. 4).

STAT3 expression following BPA and STAT3 siRNA5 treatment. To probe the interference effect and BPA-promoted STAT3 expression in MCF-7 cells, we performed a combined treatment of BPA and STAT3 siRNA5, and the expression of

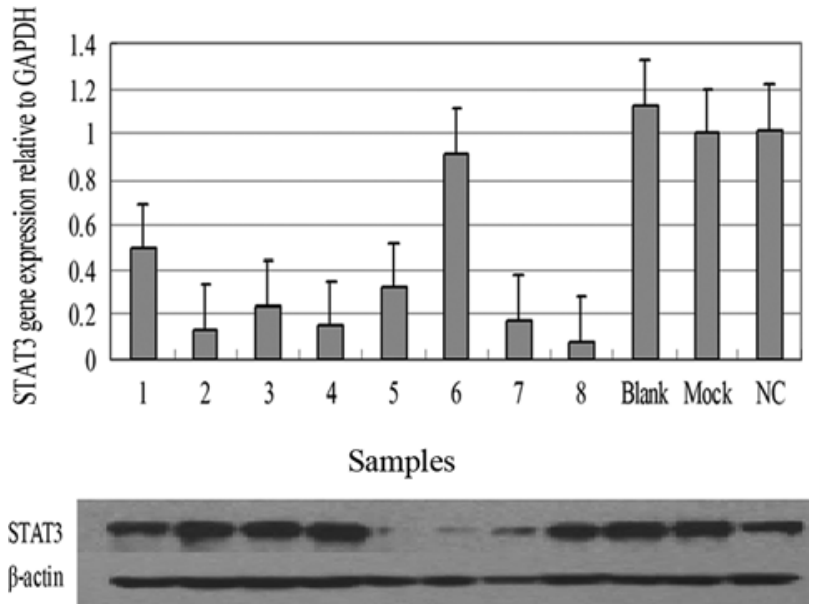

Figure 4. Expression of STAT3 of different siRNA samples designed to screen the optimal segments for transfection. The RT-PCR and Western blotting results show the expression of STAT3 after transfecting the 8 siRNA segments. Lanes from left to right: 1 , siRNA1; 2, siRNA2; 3, siRNA3; 4, siRNA4; 5, siRNA5; 6, siRNA6; 7, siRNA7; 8, siRNA8; 9, blank group; 10, mock group; 11, negative control.

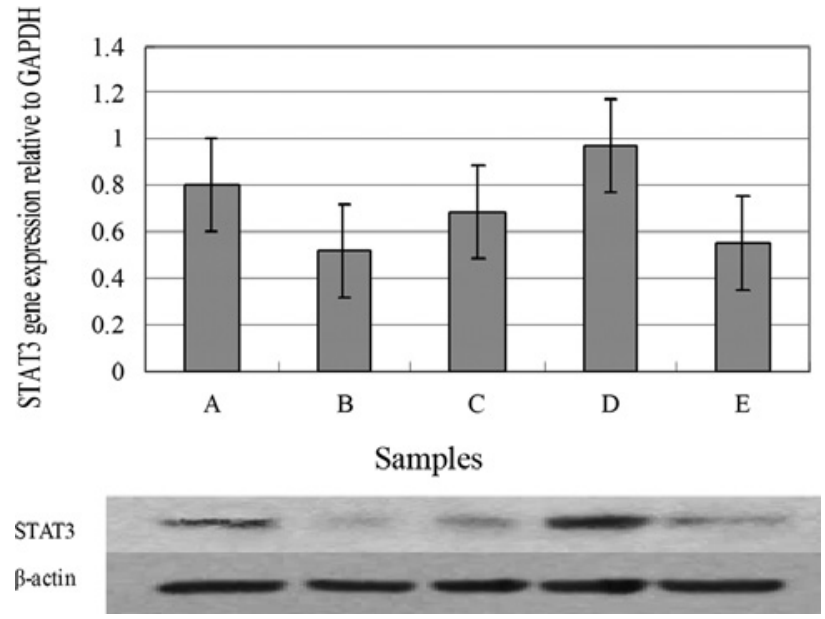

Figure 5. STAT3 expression in MCF-7 cells with or without the combined treatment of BPA and siRNA interference. A, blank group: MCF-7 cells; $\mathrm{B}$, STAT3 siRNA5; C, BPA (at the working concentration of $1 \mu \mathrm{M}$ ) + dehydrated alcohol + STAT3 siRNA5; D, BPA (at the working concentration of $1 \mu \mathrm{M})+$ dehydrated alcohol + negative control; E, dehydrated alcohol (the volume is equal to BPA, which was at working concentration) + STAT3 siRNA5.

STAT3 was detected following a 48-h treatment. As shown in Fig. 5, given the results of the RT-PCR and Western blotting, the efficiency of RNAi5 for the targeted knockdown of STAT3 is approximately that of the STAT3 siRNA5 alone treatment group (group B). As compared to the normal breast cancer cells (group A) and BPA with the negative control treatment group (group D), BPA promoted the expression of STAT3. In addition, treatment with $1 \mu \mathrm{M}$ BPA in the presence of STAT3 siRNA5 and vehicle (group C) suggests that BPA is not capable of activating STAT3 after siRNA5 activity (Fig. 5).

MTT assay following STAT3 siRNA5 and BPA treatment. To establish the relevance of STAT3 in the signaling pathway of BPA-induced cell growth, the cytotoxicity of MCF-7 cells was determined by the MTT assay following the combined treat- 


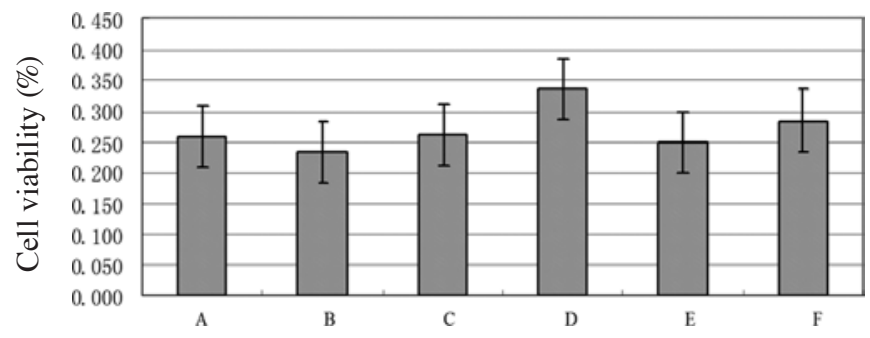

Figure 6. MCF-7 cells were treated with BPA and STAT3 siRNA5 for $48 \mathrm{~h}$ to determine the cytotoxicity by the MTT assay. A, blank group: MCF-7 cells; B, STAT3 siRNA5; C, BPA (at the working concentration of $1 \mu \mathrm{M}$ ) + dehydrated alcohol + STAT3 siRNA5; D, BPA (at the working concentration of $1 \mu \mathrm{M})+$ dehydrated alcohol + negative control; E, dehydrated alcohol (the volume is equal to BPA, which was at working concentration) + STAT3 siRNA5; F, negative control (the volume is equal to BPA that was at working concentration) group.

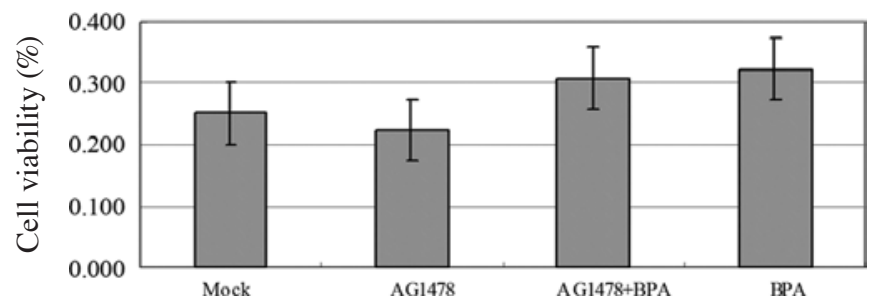

Figure 7. BPA and AG1478 were treated together to explore the effect of AG148 and BPA on EGFR. Cells were treated with BPA and AG148 for $48 \mathrm{~h}$. Cytotoxicity was determined by the MTT assay. The mock group was DMSO + dehydrated alcohol group; AG1478 group was at the working concentration of $10 \mu \mathrm{M}$; AG148 and BPA groups were at their working concentration of 10 and $1 \mu \mathrm{M}$, respectively; BPA group was at the working concentration of $1 \mu \mathrm{M}$.

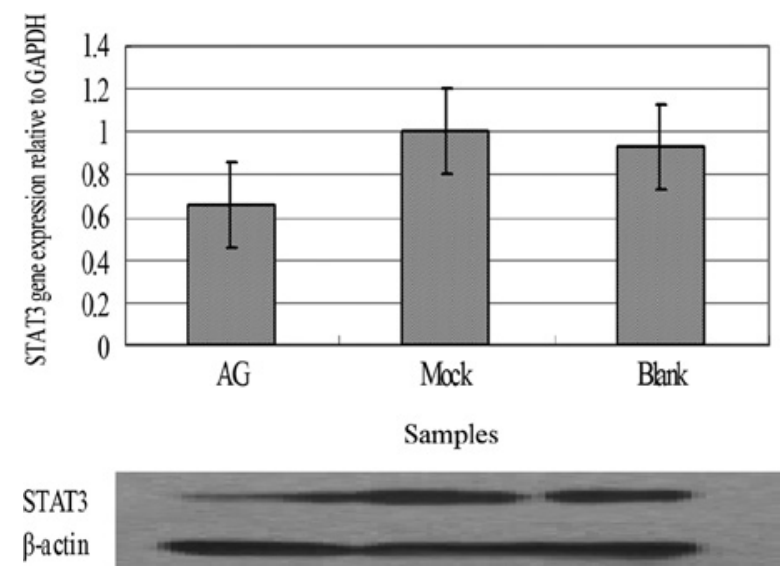

Figure 8. Cells were treated with AG148 for 48 h. MCF-7 cells were collected to assay the expression of STAT3 after blocking EGFR. AG group, STAT3 expression, which was treated with AG1478 at the working concentration of $10 \mu \mathrm{M}$; Mock group, MCF-7 cells and DMSO.

ment of BPA and STAT3 siRNA5 for $48 \mathrm{~h}$. As observed in Fig. 6, BPA with the negative control treatment group (group D) showed its proliferation effect compared to the normal breast cancer cells. However, treatment with $1 \mu \mathrm{M}$ BPA in the presence of STAT3 siRNA5 and vehicle (group C) for $48 \mathrm{~h}$ resulted in the stimulation of cell growth in MCF-7 cells similar to that of the STAT3 siRNA5 and the vehicle treatment group (group E). After STAT3 was blocked, BPA did not induce marked cell proliferation (Fig. 6).
Effect of AG1478 on BPA-induced proliferation in MCF-7 cells. To elucidate the relevance of EGFR in the signaling pathway of BPA-induced proliferation, the specific inhibitor AG1478 was pre-treated for 20 min with prior to BPA treatment. As shown in Fig. 7, treatment with AG1478 alone at a dose of $10 \mu \mathrm{M}$ did not alter the cell growth of MCF-7 cells. However, treatment with $1 \mu \mathrm{M}$ BPA in the presence of AG1478 for $48 \mathrm{~h}$ resulted in the stimulation of cell growth in MCF-7 cells similar to that of the BPA alone treatment (Fig. 7).

Effect of AG1478 and BPA on the expression of STAT3 in MCF-7 cells. To investigate whether STAT3 expression correlated to EGFR, cells were treated with AG1478 for $48 \mathrm{~h}$. The real-time PCR and Western blotting results showed an approximately 34 and $72 \%$ decrease, respectively, compared to the normal samples, which indicated that the expression of STAT3 was down-regulated after being treated with AG1478. $\mathrm{P}<0.05$ was considered to be statistically significant (Fig. 8).

\section{Discussion}

A number of investigators have indicated that BPA promotes breast cancer MCF-7 cell proliferation in the concentration range of $1 \times 10^{-7}$ to $1 \times 10^{-5} \mathrm{M}(18)$. When BPA is at nanomolar doses it often displays stronger estrogen-like activities than $\mathrm{E}_{2}$ (estradiol) itself (19). Samuelsen et al (20) confirmed that BPA often exhibits an inverted U-shaped curve, observed when treating cells with increasing doses of BPA. Cell proliferation was unchanged in the presence of $10 \mathrm{nM} \mathrm{BPA}$, increased $>40 \%$ with $100 \mathrm{nM}$ BPA, peaked with $1 \mu \mathrm{M}$ BPA and declined at higher doses. These studies are in agreement with our data, suggesting that MCF-7 cells proliferate significantly with $1 \mu \mathrm{M}$ BPA treatment for $24 \mathrm{~h}$. In addition to the optimal concentration of BPA for promoting cell proliferation, we further investigated the optimal treatment period, which is $24 \mathrm{~h}$, and the proliferation effect which may continue to $48 \mathrm{~h}$.

We also demonstrated that BPA only promotes the proliferation of ER-positive MCF-7 cells, but not of ER-negative MDA-MB-231 cells, suggesting specificity of this effect for the ER-mediated pathway. BPA induced the transcriptional activity of ERE and mimicked the effect of $E_{2}$ activity on cell proliferation (12). The specific ERs of BPA-induced proliferation remain to be clarified; however, hypotheses include: i) BPA binds differently within the ligand-binding domain of ER $\alpha$ or ER $\beta$ and recruits dissimilar coregulators (21); ii) BPA elicits rapid responses by binding to membrane-anchored ERs, or G-protein-coupled receptor 30 (GPR30) (22), and its chemoresistance is probably through the non-classical GPR30 and members of the ERR family (10); iii) BPA binds to estrogen-related receptor $\gamma(\mathrm{ERR} \gamma)$, an orphan nuclear receptor belonging to the ERR family of receptors that do not directly bind $\mathrm{E}_{2}$ (23). Moreover, Okada et al (24) have shown that BPA was found to bind at high affinity to ERR $\gamma$. Matsushima et al (25) have demonstrated that ERR $\gamma$ behaves as a constitutive activator of transcription, and that BPA binds markedly to ERR $\gamma$ (but not to ER), preserving the ERR $\gamma$ 's basal constitutive activity.

Studies have shown that BPA induced significant changes in the phosphorylation state of STAT members (26), and the effects of EEs on the phosphorylation state of transcription 
factor STATs suggest that these compounds lead to changes in gene expression secondary to the modulation of kinase/phosphatases (27). Our data have confirmed that BPA up-regulated STAT3, therefore we postulated that STAT3 is a key gene in BPA-induced cell proliferation. It has been confirmed that the effect of BPA $(10 \mu \mathrm{M})$ on stimulation of cell growth was not blocked by pretreatment with inhibitors of ERK1/2 or p38 in a dose-dependent manner, and that MAPK is unlikely to be involved in EE-induced cell growth in estrogen-responsive ovarian cancer cells (12). EGFR is the common upstream regulator of ERK1/2, p38 and STAT3; therefore, we speculated the BPA-induced cell proliferation acts through the EGFR-STAT3 pathway.

We postulated that STAT3 plays a role in BPA-induced proliferation, based on the fact that STAT3 is an oncogenic gene constitutively activated in approximately $30-60 \%$ of primary breast cancers (28). We inhibited the STAT3 gene with an RNAi technique, as it has been confirmed that STAT3 inhibition using RNA interference leads to reduced cell proliferation (29). We used STAT3 siRNA5, which markedly knocks down the expression of STAT3 with an efficiency of almost up to $100 \%$. Pretreatment by knocking down STAT3 eradicated the stimulation of cell growth by BPA in MCF-7 cells. Although there are complicated signal pathways in BPA-induced proliferation in breast cancer cells, our results indicated that the activation and transmission of STAT3 are vital elements.

In their study, Berclaz et al (13) have shown that there is a marked correlation between nuclear STAT3 and EGFR expression in breast cancer. STAT3 is the convergence of multiple oncogenic tyrosine kinase signaling pathways, such as EGFR, IL-6/JAK and Src (30). However, given that EGFR could signal to a variety of downstream effectors, the role of EGFR in BPA-promoted cell proliferation has not been fully elucidated. We explored the effect of BPA on EGFR with its inhibitor Tyrophostin AG1478 to ensure the possible signaling pathway of BPA. Tyrphostin AG1478 is an artificial micromolecular protein tyrosine kinase inhibitor, which is highly selective for EGFR (31). The in vitro experiment indicated that AG1478 was capable of blocking the EGFR signaling pathway, inhibiting proliferation of numerous tumor cells and inducing cell apoptosis (32). In addition, Tyrphostin AG1478 is a potential EGFR-targeted therapeutic agent for breast cancer (33). In our study, AG1478 did not neutralize the stimulating effect of MCF-7 cell proliferation by BPA, indicating that EGFR inhibition was not able to affect the proliferation of MCF-7 cells. Therefore, EGFR mediates negative signaling for BPA-induced breast cancer cell proliferation. In this study, we explored the relationship of ER and BPA-induced proliferation in MCF-7 cells following inhibition of EGFR, and whether ER and EGFR are non-redundant pathways remains to be further confirmed.

Our study also demonstrated that the expression of STAT3 is down-regulated after blocking EGFR, and the inhibition indicates that EGFR-STAT3 is highly correlated. It has been confirmed that the activation of EGF receptor tyrosine kinases is associated with the activation of STAT proteins (13). In addition, the recruitment sites of STAT3 within the EGFR have been identified and characterized, indicating the direct association of the two molecules (17). Notably, the combined treatment of AG1478 and siRNA inhibited STAT3 expression resulted in opposite effects on cell proliferation. Our data have demonstrated that AG1478 treatment results in an approximately $70 \%$ decrease in the expression of STAT3, and that the $30 \%$ gene expression of STAT3 is sufficient for cell proliferation, underlining the crucial role of STAT3 in cell proliferation. However, the inactivation of the STAT3 gene by siRNA acts potently and inhibits BPA-induced MCF-7 cell proliferation. Thus, STAT3 activation contributes to BPA-induced breast cancer cell proliferation.

Our study concentrated on the effect of BPA on the EGFR-STAT3 pathway in breast cancer MCF-7 cells. We confirmed that BPA increases STAT3 expression, which is a significant factor in the pathway of BPA-induced proliferation, and that STAT3 activation contributes to BPA-induced breast cancer cell proliferation. However, EGFR mediates negative signaling for BPA-induced breast cancer cell proliferation. These data emphasize a previously unrecognized signaling pathway of BPA in its carcinogenesis, thereby adding powerful support to the increasing realization of the adverse effects of BPA on human health. Given the hazard of BPA and the importance of STAT3 as a signal transducer and activator, the mechanism of STAT3-mediated BPA-induced proliferation should be further investigated.

\section{Acknowledgements}

This study was supported by the Department of Science and Technology of the Jilin Province, Project Number: 201105094.

\section{References}

1. Russo J, Tahin Q, Lareef MH, Hu YF and Russo IH: Neoplastic transformation of human breast epithelial cells by estrogens and chemical carcinogens. Environ Mol Mutagen 39: 254-263, 2002.

2. Diel P, Olff S, Schmidt S and Michna H: Effects of the environmental estrogens Bisphenol A, o, p'-DDT, p-tert- octylphenol and coumestrol on apoptosis induction, cell proliferation and the expression of estrogen sensitive molecular parameters in the human breast cancer cell line MCF-7. J Steroid Biochem Mol Biol 80: 61-70, 2002.

3. Savis DL, Bradlow HL, Barrach HJ, et al: Can environmental estrogens cause breast cancer? Sci Am 273: 167-172, 1995.

4. Le HH, Carlson EM, Chua JP and Belcher SM: Bisphenol A is released from polycarbonate drinking bottles and mimics the neurotoxic actions of estrogen in developing cerebellar neurons. Toxicol Lett 176: 149-156, 2008.

5. Dolinoy DC, Huang D and Jirtle RL: Maternal nutrient supplementation counteracts Bisphenol A-induced DNA hypomethylation in early development. Proc Natl Acad Sci USA 104: 13056-13061, 2007.

6. Weng YI, Hsu PY, Liyanarachchi S, et al: Epigenetic influences of low-dose Bisphenol A in primary human breast epithelial cells. Toxicol Appl Pharmacol 248: 111-121, 2010.

7. Wolff MS, Teitelbaum SL, Windham G, et al: Pilot study of urinary biomarkers ophytoestrogens, phthalates, and phenols in girls. Environ Health Perspect 115: 116-121, 2007.

8. Welshons WV, Nagel SC and vom Saal FS: Large effects from small exposures. III. Endocrine mechanisms mediating effects of Bisphenol A at levels of human exposure. Endocrinology 147: S56-S69, 2006.

9. Kurosawa T, Hiroi $\mathrm{H}$, Tsutsumi $\mathrm{O}$, et al: The activity of Bisphenol A depends on both the etrogens receptor subtype and the cell type. Endocr J 49: 465-471, 2002.

10. Lapensee EW, Tuttle TR, Fox SR and Ben-Jonathan N: Bisphenol $\mathrm{A}$ at low nanomolar doses confers chemoresistance in estrogen receptor- $\alpha$-positive and-negative breast cancer cells. Environ Health Perspect 117: 175-180, 2009. 
11. Jaattela M, Benedict M, Tewari M, Shayman JA and Dixit VM: $\mathrm{Bcl}-\mathrm{x}$ and Bcl-2 inhibit TNF and Fas-induced apoptosis and activation of phospholipase A2 in breast carcinoma cells. Oncogene 10: 2297-2305, 1995.

12. Park SH, Kim KY, An BS, Choi JH, Jeung EB, Leung PC and Choi KC: Cell growth of ovarian cancer cells is stimulated by xenoestrogens through an estrogen-dependent pathway, but their stimulation of cell growth appears not to be involved in the activation of the mitogen-activated protein kinases ERK-1 and p38. J Reprod Dev 55: 23-29, 2009.

13. Berclaz G, Altermatt HJ, Rohrbach V, Siragusa A, Dreher E and Smith PD: EGFR dependent expression of STAT3 (but not STAT1) in breast cancer. Int J Oncol 19: 1155-1160, 2001.

14. Lo HW, Hsu SC, Ali-Seyed M, et al: Nuclear interaction of EGFR and STAT3 in the activation of the iNOS/NO pathway. Cancer Cell 7: 575-589, 2005.

15. Hynes NE and MacDonald G: Erb $\beta$ receptors and signaling pathways in cancer. Curr Opin Cell Biol 21: 177-184, 2009.

16. Gusterson BA and Hunter KD: Should we be surprised at the paucity of response to EGFR inhibitors? Lancet Oncol 10: 522-527, 2009

17. Shao H, Cheng HY, Cook RG and Tweardy DJ: Identification and characterization of signal transducer and activator of transcription 3 recruitment sites within the epidermal growth factor receptor. Cancer Research 63: 3923-3930, 2003.

18. Suzuki T, Nakagawa Y, Takano I, Yaguchi K and Yasuda K Environmental fate of Bisphenol A and it s biological metabolites in river water and their xeno-estrogenic activity. Environ Sci Technol 38: 2389-2396, 2004.

19. Hugo ER, Brandebourg TD, Woo JG, Loftus J, Alexander JW and Ben-Jonathan N: Bisphenol A at environmentally relevant doses inhibits adiponectin release from human adipose tissue explants and adipocytes. Environ Health Perspect 116: 1642-1647, 2008.

20. Samuelsen M, Olsen C, Holme JA, Meussen-Elholm E, Bergmann A and Hongslo JK: Estrogen-like properties of brominated analogs of Bisphenol A in the MCF-7 human breast cancer cell line. Cell Biol Toxicol 17: 139-151, 2001.

21. Safe SH, Pallaroni L, Yoon K, Gaido K, Ross S and McDonnell D: Problems for risk assessment of endocrine-active estrogenic compounds. Environ Health Perspect 10: 925-929, 2002.

22. Watson CS, Bulayeva NN, Wozniak AL and Finnerty CC: Signaling from the membrane via membrane estrogen receptor-alpha: estrogens, xenoestrogens, and phyto-estrogens Steroids 70: 364-371, 2005.
23. Ariazi EA and Jordan VC: Estrogen-related receptors as emerging targets in cancer and metabolic disorders. Curr Top Med Chem 6: 203-215, 2006

24. Okada H, Tokunaga T, Liu X, Takayanagi S, Matsushima A and Shimohigashi Y: Direct evidence revealing structural elements essential for the high binding ability of Bisphenol A to human estrogen-related receptor- $\gamma$. Environ Health Perspect 116: 32-38, 2008.

25. Matsushima A, Kakuta Y, Teramoto T, et al: Structural evidence for endocrine disruptor Bisphenol A binding to human nuclear receptor ERR gamma. J Biochem 142: 517-524, 2007.

26. Canesi L, Betti M, Lorusso LC, Ciacci C and Gallo G: 'In vivo' effects of Bisphenol A in Mytilus hemocytes: modulation of kinase-mediated signalling pathways. Aquat Toxicol 71: 73-84, 2005.

27. Canesi L, Lorusso LC, Ciacci C, Betti M, Zampini M and Gallo G: Environmental estrogens can affect the function of mussel hemocytes through rapid modulation of kinase pathways. Gen Comp Endocrinol 38: 58-69, 2004.

28. Hatake K, Tokudome N and Ito Y: Next generation molecular targeted agents for breast cancer: focus on EGFR and VEGFR pathways. Breast Cancer 14: 132-149, 2007.

29. Madoux F, Koenig M, Nelson E, et al: Modulators of STAT transcription factors for the targeted therapy of cancer (STAT3 activators). Bethesda (MD): National Center for Biotechnology Information 16: 2-10, 2010.

30. Niu G, Wright KL, Huang M, et al: Constitutive STAT3 activity up-regulates VEGF expression and tumor angio-genesis. Oncogene 21: 2000-2008, 2002.

31. El-Marjou A, Delouvée A, Thiery JP and Radvanyi F: Involvement of epidermal growth factor receptor in chemically induced mouse bladder tumor progression. Carcinogenesis 21: 2211-2218, 2000.

32. Ellis AG, Nice EC, Weinstock J, Levitzki A, Burgess AW and Webster LK: High-performance liquid chromatographic analysis of the tyrphostin AG1478, a specific inhibitor of the epidermal growth factor receptor tyrosine kinase, in mouse plasma. J Chromatogr B Biomed Sci Appl 754: 193-199, 2001.

33. Zhang YG, Du Q, Fang WG, Jin ML and Tian XX: Tyrphostin AG1478 suppresses proliferation and invasion of human breast cancer cells. Int J Oncol 33: 595-602, 2008 